\title{
A simple and efficient purification method of native immunoreactive antigen for diagnosis of camel hydatidosis
}

\author{
Nagwa I. Toaleb ${ }^{1}$, Mohamed S. Helmy르 Eman E. El Shanawany ${ }^{1}$ and Eman H. Abdel-Rahman ${ }^{1}$
}

1. Department of Parasitology and Animal Diseases, National Research Centre, El Buhouth St., Dokki, Cairo, Egypt; 2. Department of Molecular Biology, National Research Centre, El Buhouth St., Dokki, Cairo, Egypt.

Corresponding author: Nagwa I. Toaleb, e-mail: nagwaibrahim26@yahoo.com

Co-authors: MSH: mohamedhelmy82@yahoo.com, EEE: ee.elshanawany@hotmail.com, EHA: emanhussein1@hotmail.com

Received: 06-08-2019, Accepted: 09-12-2019, Published online: 23-01-2020

doi: www.doi.org/10.14202/vetworld.2020.141-146 How to cite this article: Toaleb NI, Helmy MS, El Shanawany EE, Abdel-Rahman EH (2020) A simple and efficient purification method of native immunoreactive antigen for diagnosis of camel hydatidosis, Veterinary World, 13(1): 141-146.

\begin{abstract}
Background: Cystic echinococcosis (CE), a zoonotic disease that affects animal and human health, is of increasing economic importance due to high morbidity rates and high economic losses in the livestock industry.
\end{abstract}

Aim: The present study was conducted to purify the antigen from hydatid cyst fluid (HCF) with high diagnostic efficacy of camel hydatidosis using indirect enzyme-linked immunosorbent assay (ELISA).

Materials and Methods: The HCF antigen was purified using Sephacryl S-300 column chromatography. Characterization of fractions was performed using reducing and non-reducing sodium dodecyl sulfate-polyacrylamide gel electrophoresis (SDS-PAGE) and Western blot analysis. Further, antibodies against Echinococcus granulosus cysts in camel serum were detected using indirect ELISA.

Results: The purification process resulted in three fractions of antigens: FI, FII, and FIII. Indirect ELISA showed that higher diagnostic efficacy was observed in FI than in FII and FIII. Indirect ELISA, in which FI was utilized, showed 88\% sensitivity and $91.7 \%$ specificity. Non-reducing SDS-PAGE showed that FI had two bands of molecular weights 120 and $60 \mathrm{kDa}$. Western blot analysis of FI demonstrated that 60,38 , and $22 \mathrm{kDa}$ were antigenic bands when reacted with naturally infected camel sera with E. granulosus cysts. Using indirect ELISA, F1 recorded an infection percentage of $81.7 \%$ in randomly collected camel serum samples.

Conclusion: FI is a promising antigen for accurate diagnosis of camel CE using indirect ELISA.

Keywords: camel hydatidosis, cystic echinococcosis, Echinococcus granulosus, gel filtration chromatography, hydatid cyst fluid, indirect enzyme-linked immunosorbent assay, sodium dodecyl sulfate-polyacrylamide gel electrophoresis, Western blot.

\section{Introduction}

Cystic echinococcosis (CE), a zoonotic disease caused by the larval stage of the Echinococcus granulosus, is an animal and human health problem of increasing economic and public health importance [1]. CE in animals is asymptomatic and its diagnosis is made at necropsy [2,3], which depends on the analysis of morphological features using light or electron microscopy $[4,5]$. The serological diagnosis of CE improves the quality of the management and treatment of the disease. Enzyme-linked immunosorbent assay (ELISA) has been useful for the diagnosis of CE in domestic animals and humans [6]. It is generally cheap, quick, and requires fewer trained and specialized personnel [7-9].

Commercially available immunodiagnostic kits often depend on crude antigen preparations of

Copyright: Toaleb, et al. Open Access. This article is distributed under the terms of the Creative Commons Attribution 4.0 International License (http://creativecommons.org/licenses/by/4.0/), which permits unrestricted use, distribution, and reproduction in any medium, provided you give appropriate credit to the original author(s) and the source, provide a link to the Creative Commons license, and indicate if changes were made. The Creative Commons Public Domain Dedication waiver (http://creativecommons.org/ publicdomain/zero/1.0/) applies to the data made available in this article, unless otherwise stated.
E. granulosus hydatid cyst fluid (HCF); hence, it lacks satisfactory specificity and sensitivity [10]. Unsatisfactory performances may be due to the poor quality of antigen preparations. To avoid this problem, novel tests using purified antigens have been utilized in previous studies [11,12]. Purification of HCF antigens is essential to remove cross-reactivity and increase the sensitivity of techniques for the detection of low levels of antibodies [13]. El Deeb et al. [14] reported that the detected antibody of E. granulosus HCF in sheep using different antigens showed that purified HCF antigen was the most effective antigen compared with excretory/secretory and somatic antigens of protoscolex. The response of HCF antigens depends on the host and the location of the parasitic cysts [10]. Furthermore, the specificity and diagnostic efficacy of purified HCF antigen were higher than those of protoscolex antigen in serological studies on $\mathrm{CE}$ among camels in Egypt [15].

Antigen $\mathrm{B}$ and antigen 5, the most immunogenic antigen among $\mathrm{HCF}$ antigens, play an important role in the life cycle of the cestode [16]. However, interestingly, antigen 5 is immunoreactive in all stages of CE pathology compared with antigen $\mathrm{B}$, which 
reveals a reduced antibody capturing activity in all CE stages [17]. Moreover, antigen 5 is one of the most immunogenic proteins present in HCF. Pagnozzi et al. [18] obtained an HCF fraction highly enriched in native antigen 5 through fast protein liquid chromatography on a Superdex-200 column with strong reactivity in both ELISA and Western blotting assays. The enriched antigen 5 fraction obtained from concentrated aliquots of sheep HCF was used for the accurate diagnosis of human CE using ELISA [19].

This study aimed to adopt a simple and inexpensive HCF purification method to obtain purified antigen of high diagnostic potency for camel CE.

\section{Materials and Methods}

\section{Ethical approval}

All experimental procedures were performed according to the institutional guidelines of the National Research Centre's Animal Research Committee under protocol number 18/198.

\section{Antigen preparation}

The HCF antigen was prepared from hydatid cysts removed from the lungs of slaughtered camels in an abattoir in Cairo in accordance with Swarna and Parija [20]. Briefly, HCF was aseptically aspirated in a sterile tube. $\mathrm{HCF}$ was further centrifuged at $6000 \times \mathrm{g}$ for $45 \mathrm{~min}$ at $4^{\circ} \mathrm{C}$ and then dialyzed against phosphate buffer saline with $\mathrm{pH}$ 7.2. Finally, HCF was centrifuged at $6000 \times \mathrm{g}$ for $30 \mathrm{~min}$. The supernatant was collected and its protein content was estimated using the Lowry et al. method [21].

\section{Preparation of hyperimmune rabbit serum}

Antibodies against E. granulosus HCF antigen were produced in two healthy male New Zealand rabbits free of parasitic infections, weighing $1.5 \mathrm{~kg}$, and about 2 months of age. Two rabbits were subcutaneously immunized with $40 \mu \mathrm{g} / \mathrm{kg}$ of crude HCF antigen emulsified in Freund's complete adjuvant according to Guobadia and Fagbemi [22]. On day 14, another dose of antigen was injected in Freund's incomplete adjuvant according to Fagbemi et al. [23]. The second and third booster doses were administered on days 21 and 28 , respectively. After 4 days, the last blood samples were collected from the ear vein and serum samples were separated.

\section{Collection of serum samples}

In total, 25 positive camel serum samples were collected from infected slaughtered camels with lung cysts during several visits to a local abattoir. Further, 12 blood samples were collected as negative samples from healthy camels free of cysts as confirmed by veterinary inspection. ELISA was used to diagnose 93 camel serum samples randomly collected from a local abattoir in Cairo. Sera were separated from all blood samples and kept at $-20^{\circ} \mathrm{C}$ until use.

\section{Purification of HCF antigen by gel filtration}

The concentrated solution containing the HCF antigen was applied onto a Sephacryl S-300 gel filtration column chromatography $(142 \mathrm{~cm}$ in length and
$1.75 \mathrm{~cm}$ in diameter). The column was equilibrated and fractions were eluted with $0.02 \mathrm{~mol} / \mathrm{l}$ phosphate buffer saline ( $\mathrm{pH} 7.2$ ) containing $0.03 \mathrm{~mol} / \mathrm{l}$ phenylmethylsulfonyl fluoride and $0.02 \% \mathrm{NaN}_{3}$ at a flow rate of $30 \mathrm{ml} / \mathrm{h}$. The eluted fractions were collected in 175 tubes of $2 \mathrm{ml}$ each. Furthermore, the proteins were monitored by measuring the absorbance at 280 $\mathrm{nm}$ using a spectrophotometer (Shimadzu).

\section{ELISA}

Diagnostic value of crude antigen and isolated fractions was compared using indirect ELISA with hyperimmune rabbit sera and naturally infected camel sera according to Santiago and Hillyer [24]. The most potent fraction was adopted to diagnose $E$. granulosus cysts in randomly collected camel sera. Checkerboard titration was used to determine the antigen concentrations and dilution of sera as well as protein A horseradish peroxidase (Sigma Chem. Co., St. Louis, USA). The cutoff values were measured as mean values +3SD [25].

\section{Characterization of fractions}

Non-reducing sodium dodecyl sulfate-polyacrylamide gel electrophoresis (SDS-PAGE) was performed using $12 \%$ polyacrylamide gel [26] stained with silver stain [27], photographed, and analyzed using Molecular Imager Gel Doc ${ }^{\mathrm{TM}} \mathrm{XR}+$ with Image Lab Software (Bio-Rad, California, USA). Molecular weights of bands observed were calculated using molecular weight of standard proteins which were electrophoresed on the same gel.

\section{Immunoblot}

After another electrophoresis, under reducing condition in $10 \%$ SDS-PAGE, eluted fractions, crude HCF antigens, and Prestained Protein Ladder (Vivantis Technologies) were blotted onto nitrocellulose membrane as described by Towbin et al. [28] in a blotting system. After washing and blocking, the membrane was incubated with diluted naturally infected camel sera (1:200). Protein A horseradish peroxidase conjugate was used at a dilution of 1:2000. In addition, bands were observed by adding 4-chloro-1-naphthol (Sigma), and the membrane was photographed by Molecular Imager Gel Doc ${ }^{\mathrm{TM}} \mathrm{XR}+$ with Image Lab Software.

\section{Statistical analysis}

Sensitivity and specificity were calculated according to Gonzalez-Sapienza et al. [29], and the diagnostic parameters used were as follows: Truepositive values (Tp), sera from camel naturally infected with E. granulosus as confirmed by parasitological examination; false-negative values (Fn), sera from camel infected with CE showing negative readings; false-positive values ( $\mathrm{Fp}$ ), sera from non-infected camels showing a positive result; and true-negative values (Tn), sera from healthy camels free of cysts as confirmed by veterinary inspection showing negative readings. 


\section{Results}

\section{Isolated fractions}

The purification process resulted in the isolation of three fractions of antigens: FI, FII, and FIII (Figure-1). The protein content of the fractions FI, FII, and FIII was 54.6, 38.7, and $69.6 \mathrm{mg} / \mathrm{ml}$, respectively.

\section{Electrophoretic profile of the isolated fractions}

FI migrates in two bands; a non-complex band with a molecular weight of $120 \mathrm{kDa}$ and a complex band with a molecular weight of $60 \mathrm{kDa}$ under non-reducing conditions in 12\% SDS-PAGE. Conversely, FII revealed a complex band with molecular weight of $72 \mathrm{kDa}$ and FIII revealed a complex band molecular weight of $74 \mathrm{kDa}$ (Figure-2).

\section{Diagnostic potency of the three purified fractions}

Serum samples from naturally infected camels with E. granulosus and hyperimmune rabbit sera showed a strong reaction against these three fractions. Higher potency in the detection of antibodies against CE was observed in purified FI than in FII and FIII, as shown in Figure-3.

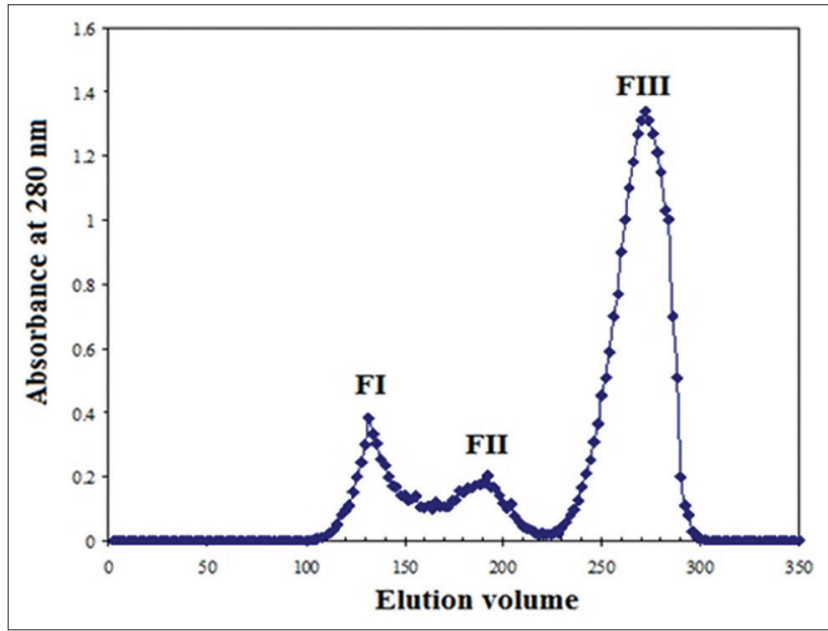

Figure-1: Purification profile of hydatid cyst fluid antigen on Sephacryl S 300 column chromatography.

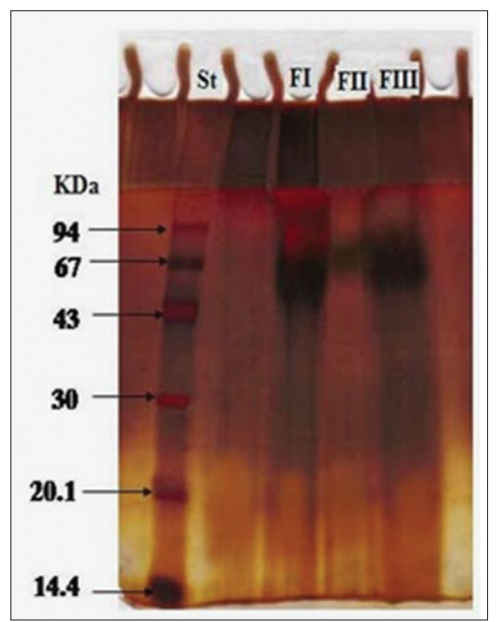

Figure-2: Electrophoretic profile of the three fractions of camel HCF antigen FI, FII, FIII, and Low Molecular weight standards (lane St).
Sensitivity, specificity, positive predictive value (PPV), and negative predictive value (NPV) of FI using indirect ELISA

Using ELISA, sensitivity of purified FI antigen against positive naturally infected camel serum samples was found to be $88 \%$, and specificity was 91.7\%. FI had PPV of $95.6 \%$ and NPV of $78.6 \%$.

\section{Diagnosis of camel CE}

Indirect ELISA was adopted to diagnose E. granulosus cysts in camel using purified FI as an antigen. FI antigen showed that $81.7 \%$ of randomly selected camel serum samples were infected with $\mathrm{CE}$, as shown in Figure-4.

\section{Antigenic bands}

The antigenic bands in crude HCF antigen were $120,74,60,57,38,22$, and $8 \mathrm{kDa}$ (Figure-5 Lane B). The antigenic bands of FI antigen were identified as 60,38 , and $22 \mathrm{kDa}$ using immunoblot under reducing condition, in which infected camel serum samples were utilized (Figure-5 Lane A).

\section{Discussion}

In the present study, Sephacryl S-300 column chromatography was used to purify the HCF antigen

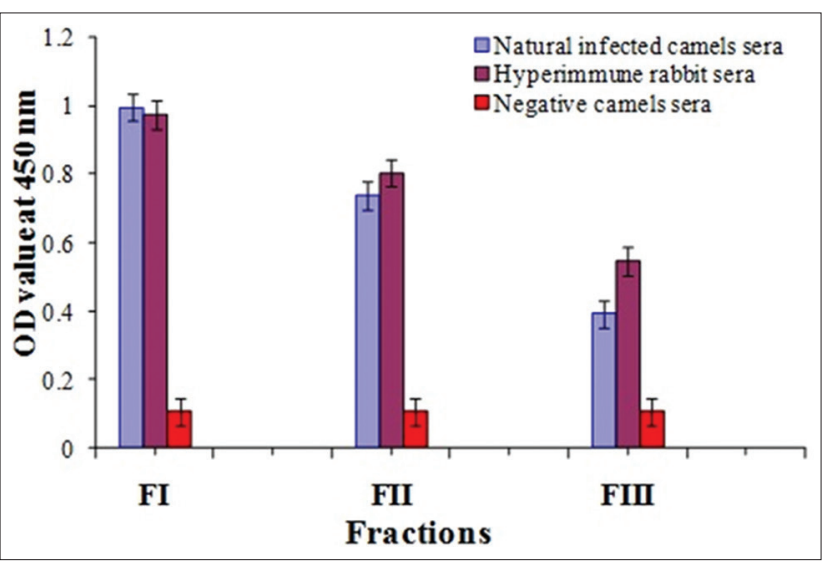

Figure-3: Comparative diagnostic potency of FI, FII and FIII.

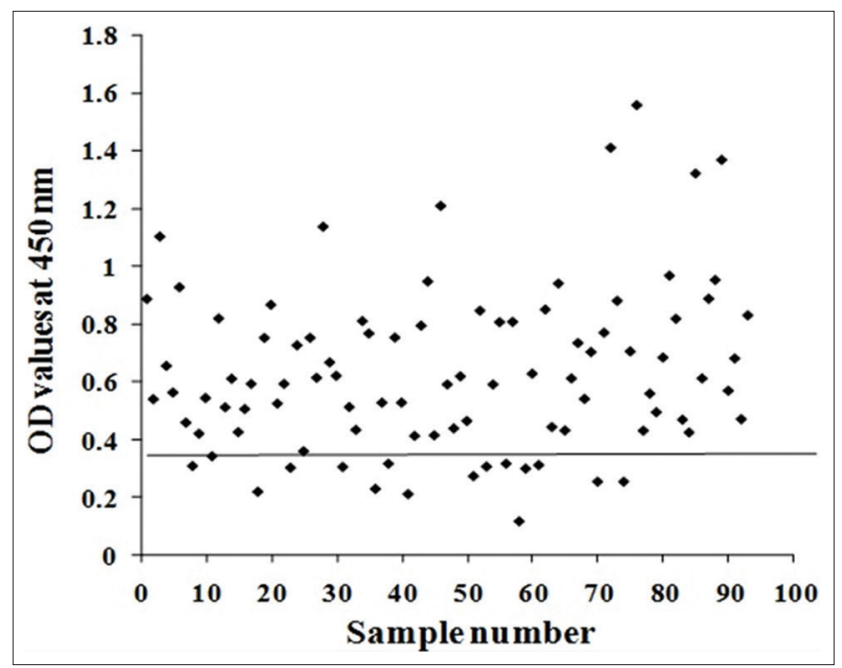

Figure-4: Diagnostic potency of FI in collected camel random serum samples. 


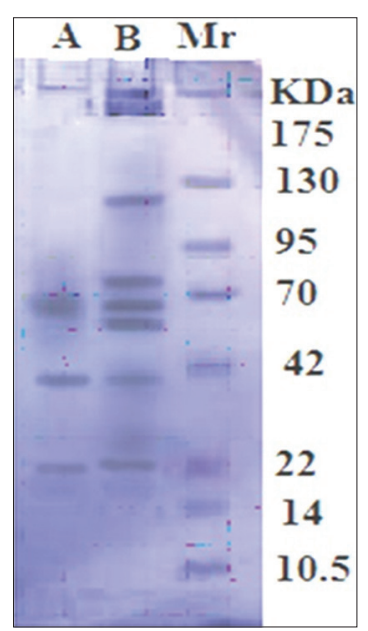

Figure-5: Antigenic bands under reducing condition recognized by naturally infected camel serum in crude HCF antigen (Lane B) and purified FI (Lane A). Standard's molecular weight (Lane Mr).

collected from camel lung cysts. Three fractions, FI, FII, and FIII, were isolated. Indirect ELISA showed that FI was the most potent diagnostic antigen. This antigen contained two bands, a non-complex band with a high molecular weight of $120 \mathrm{kDa}$ and a complex band with a molecular weight of $60 \mathrm{kDa}$, as confirmed using SDS-PAGE under non-reducing conditions. The previous studies recorded antigen 5 and antigen $\mathrm{B}$ as the most potent diagnostic antigens of camel E. granulosus cysts [30,31]. Antigen 5 has a high molecular weight of $400 \mathrm{kDa}$ and dissociates into complex components of $60-70 \mathrm{kDa}$ under reducing conditions $[1,11]$. This behavior is similar to that of FI, which showed a band with a high molecular weight of $120 \mathrm{kDa}$ that dissociated into three antigenic bands at molecular weights of 60,38 , and $22 \mathrm{kDa}$ under reducing conditions by immunoblotting with positive $\mathrm{CE}$ camel sera.

The appearance of these bands was possibly due to the dissociation of the high molecular band into a small band of $60 \mathrm{kDa}$ and two smaller bands of 38 and $22 \mathrm{kDa}$ under reducing condition. This result may be in agreement with that of a previous study by Monteiro et al. [32], which suggests that native antigen 5 complex has a large molecule of $600 \mathrm{kDa}$ equivalent to an oligomeric structure of about 10 units. Correspondingly, antigen 5 is an oligomeric thermolabile glycoprotein that migrates as 57 and $67 \mathrm{kDa}$ bands in SDS-PAGE under non-reducing conditions and as 38 and $22 \mathrm{kDa}$ bands under reducing conditions [33]. Pagnozzi et al. [18] found that antigen 5 purified by fast protein liquid chromatography, analyzed by SDS-PAGE under non-reducing and reducing conditions, and examined by immunoblotting with positive CE patient sera yields reduced antigen 5 with $38 \mathrm{kDa}$ band of light reactivity and non-reduced antigen 5 with $60 \mathrm{kDa}$ band, which has the strongest reactivity. The $22 \mathrm{kDa}$ antigenic band of antigen 5 results due to the presence of heparin sulfate proteoglycans binding sites [34,35]. These observations led to the comparison of FI with antigen 5 where both had high-molecular-weight complex dissociating into smaller entities of diagnostic potency of camel CE. In the current study, indirect ELISA was used for serodiagnosis of camel E. granulosus cysts recording $88 \%$ sensitivity and $91.7 \%$ specificity. These results matched those of a previous study by Pagnozzi et al. [19], which reported that antigen 5 ELISAs revealed different sensitivity $(88.3 \%)$ without significant differences in specificity $(92.5 \%)$.

The origin of FI from camel cyst is an additional advantage of FI. Based on molecular identification studies of animal and human isolates, camel cysts are more potent in causing infection in humans [36,37]. The Egyptian G6 strain nucleotide sequence was the predominant genotype in Egypt, and its involvement has been shown by several studies [38-40].

\section{Conclusion}

The diagnostic antigen of camel origin can be successfully utilized for the diagnosis of $E$. granulosus cysts by ELISA, which may help control the infection and minimize transmission to humans. In addition, the antigen of camel origin could successfully be utilized in the diagnosis of human infection. A local HCF antigen FI isolated by an easy, one-step, low-cost purification method proved to exhibit high diagnostic potency for camel CE.

\section{Authors' Contributions}

All authors participated in the study design. NIT collected lung hydatid cysts and blood samples from slaughtered camels, prepared crude antigen, performed a hyperimmune rabbit serum and carried out Western blot. MSH performed a purification of HCF antigen by Sephacryl S-300 gel filtration column chromatography. MSH and EEE carried out SDSPAGE under non-reducing conditions. NIT, EEE and EHA shared in ELISA for the detection of CE-specific antibodies. NIT and EHA analyzed the data. NIT and EHA participated in writing the manuscript. All authors revised and approved the final manuscript.

\section{Acknowledgments}

National Research Centre, Egypt is greatly appreciated for funding this work, Agreement No. 11020201.

\section{Competing Interests}

The authors declare that they have no competing interests.

\section{Publisher's Note}

Veterinary World remains neutral with regard to jurisdictional claims in published institutional affiliation.

\section{References}

1. Deplazes, P., Rinaldi, L., Alvarez Rojas, C.A., Torgerson, P.R., Harandi, M.F., Romig, T., Antolova, D., 
Schurerxx, J.M., Lahmarjjjj, S., Cringolix, G., Magambo, J., Thompson, R.C.A. and Jenkinsx, E.J. (2017) Global distribution of alveolar and cystic echinococcosis. Adv. Parasitol., 95: 315-493.

2. Kern, P., da Silva, A.M., Akhan, O., Müllhaupt, B., Vizcaychipi, K.A., Budke, C. and Vuitton D.A. (2017) The echinococcosis: Diagnosis, clinical management and burden of disease. Adv. Parasitol., 96: 259-369.

3. Craig, P., Mastin, A., Kesteren, F. and Boufana, B.B. (2015) Echinococcus granulosus: Epidemiology and state-ofthe-art of diagnostics in animals. Vet. Parasitol., 213(3-4): 132-148.

4. Arbabi, M., Pirestani, M., Delavari, M., Hooshyar, H., Abdoli, A. and Sarvi, S. (2017) Molecular and morphological characterizations of Echinococcus granulosus from human and animal isolates in Kashan, Markazi province, Iran. Iran J. Parasitol., 12(2): 177-187.

5. La-Rocca, S., Farias, J., Chalar, C., Kun, A.E. and Fernandez, V. (2019) Echinococcus granulosus: Insights into the protoscolex F-actin cytoskeleton. Acta Trop., 199: 1051222.

6. Bauomi, I.R., El-Amir, A.M., Fahmy, A.M., Zalat, R.S. and Diab, T.M. (2015) Evaluation of purified $27.5 \mathrm{kDa}$ protoscolex antigen-based ELISA for the detection of circulating antigens and antibodies in sheep and human hydatidosis. J. Helminthol.,89(5): 577-583.

7. Zhang, W., Wen, H., Li, J., Lin, R. and McManus, D.P. (2012) Immunology and immunodiagnosis of cystic echinococcosis: An update. Clin. Dev. Immunol., 2012: 10, Article ID 101895.

8. Sunita, T., Khurana, S., Malla, N. and Dubey, M.L. (2011) Immunodiagnosis of cystic echinococcosis by antigen detection in serum, urine, and saliva samples. Trop. Parasitol., 1(1): 33-38.

9. Bashiri, S., Mansoor, F.N. and Valadkhani, Z. (2019) Expansion of a highly sensitive and specific ELISA test for diagnosis of hydatidosis using recombinant $\mathrm{EgB} 8 / 2$ protein. Iran. J. Basic. Med. Sci., 22(2): 134-139.

10. Sbihi, Y., Rmiqui, A., Rodriguez-Cabezas, M.N., Orduña, A., Rodriguez-Torres, A. and Osuna, A. (2001) Comparative sensitivity of six serological tests and diagnostic value of ELISA using purified antigen in hydatidosis. J. Clin. Lab. Anal., 15(1): 14-18.

11. Golassa, L., Abebe, T. and Hailu, A. (2011) Evaluation of crude hydatid cyst fluid antigens for the serological diagnosis of hydatidosis in cattle. J. Helminthol., 85(1): 100-108.

12. Toaleb, N.I., Derbala, A.A. and Abdel-Rahman, E.H. (2011) Comparative diagnostic evaluation of crude and isolated fractions of Echinococcus granulosus in dogs. Glob. Vet., 7(6): 587-592.

13. El Shanawany, E.E., Toaleb, N.I. and Rahman, E.H.A. (2019) Hydatid cyst germinal layer purified glycoproteins for diagnosis of camel cystic echinococcosis. Int. J. Vet. Sci., 8(2): 101-105.

14. El Deeb, S., Aly, I., Mahna, N., Faried, A., Zalat, R. and Younis, M. (2017) Purification and characterization of Echinococcus granulosus cathepsin-B protein and evaluation of its role as a diagnostic marker. Glob. Vet., 18(2): 137-145.

15. Mousa, W.M., Mahdy, O.A., Abdel-Wahab, A.M. and El-Gameel, O.A. (2015) Epidemiological and serological studies on cystic echinococcosis among camels in Egypt. J. Parasitol. Photon., 105(3729-2384): 212-218.

16. Díaz, A., Casaravilla, C., Barrios, A.A. and Ferreira, A.M. (2016) Parasite molecules and host responses in cystic echinococcosis. Parasite Immunol., 38(3): 193-205.

17. Ahn, C.S., Han, X., Bae, Y.A., Ma, X., Kim, J.T., Cai, H., Yang, H.J., Kang, I., Wang, H. and Kong, Y. (2015) Alteration of immunoproteome profile of Echinococcus granulosus hydatid fluid with progression of cystic echinococcosis. Parasit. Vectors., 8: 10.

18. Pagnozzi, D., Biosa, G., Addis, M.F., Mastrandrea, S.,
Masala, G. and Uzzau, S. (2014) An easy and efficient method for native and immunoreactive Echinococcus granulosus antigen 5 enrichment from hydatid cyst fluid. PLoS One, 9(8): 1-12.

19. Pagnozzi, D., Addis, M.F., Biosa, G., Roggio, A.M., Tedde, V., Mariconti, M., Tamarozzi, F., Meroni, V., Masu, G., Masala, G., Brunetti, E. and Uzzau, S. (2016) Diagnostic accuracy of antigen 5-based ELISAs for human cystic echinococcosis. PLoS. Negl. Trop. Dis., 10(3): 1-14.

20. Swarna, S. and Parija, S.C. (2008) Dot-ELISA for evaluation of hydatid cyst wall, protoscoleces and hydatid cyst fluid antigens in the serodiagnosis of cystic echinococcosis. Rev. Inst. Med. Trop. São. Paulo., 50(4): 233-236.

21. Lowry, O.H., Rosebrough, N.J., Farr, A.L. and Randall, R.J. (1951) Protein measurement with the folin phenol reagent. J. Biol. Chem., 193(1): 265-275.

22. Guobadia, E.E. and Fagbemi, B.O. (1997) The isolation of Fasciola gigantica-specific antigens and their use in the serodiagnosis of fascioliasis in sheep by the detection of circulating antigens. Vet. Parasitol., 68(3): 269-282.

23. Fagbemi, B.O., Obarisiaghon, I.O. and Mbuh, J.V. (1995) Detection of circulating antigen in sera of Fasciola gigantica infected cattle with antibodies reactive with a Fasciolaspecific $88 \mathrm{kDa}$ antigen. Vet. Parasitol., 58(3): 235-246.

24. Santiago, N. and Hillyer, G.V. (1988) Antibody profiles by EIIB and ELISA of cattle and sheep infected with Fasciola hepatica. J. Parasitol., 74(5): 810-818.

25. Jin, Y., Anvarov, K., Khajibaev, A., Hong, S. and Hong, S. (2013) Serodiagnosis of echinococcosis by ELISA using cystic fluid from Uzbekistan sheep. Korean J. Parasitol., 51(3): 313-317.

26. Laemmli, U.K. (1970) Cleavage of structural protein during assembly of the head of bacteriophage T. Nature, 27(5259): 680-685.

27. Wray, W., Boulikas, T., Wray, V.P. and Hancok, R. (1981) Silver staining of proteins in polyacrylamide gel. Anal. Biol. Chem., 118(4): 197-203.

28. Towbin, H., Staehelin, T. and Gordon, J. (1979) Electrophoretic transfer of proteins from polyacrylamide gels to nitrocellulose sheets: Procedure and some applications. Proc. Nat. Acad. Sci., 76(9): 4350-4354.

29. Gonzalez-Sapienza, G., Lorenzo, C. and Nieto, A. (2000) Improved immunodiagnosis of cystic hydatid disease by using a synthetic peptide with higher diagnostic value than that of its parent protein, Echinococcus granulosus antigen B. J. Clin. Microbiol., 38(11): 3979-3983.

30. Ortona, E., Rigano, R., Margutti, P., Notargiacomo, S., Ioppolo, S., Vaccari, S., Barca, S., Buttari, B., Profumo, E., Teggi, A. and Siracusano, A. (2000) Native and recombinant antigens in the immunodiagnosis of human cystic echinococcosis. Parasite Immunol., 22(11): 553-559.

31. Pagnozzi, D., Addis, M.F., Biosa, G., Roggio, A.M., Tedde, V., Mariconti, M., Tamarozzi, F., Meroni, V., Masu, G., Masala, G., Brunetti, E. and Uzzau, S. (2016) Diagnostic accuracy of antigen 5-based ELISAs for human cystic echinococcosis. PLoS. Negl. Trop. Dis., 10(3): e0004585.

32. Monteiro, K.M., Cardoso, M.B., Follmer, C., da Silveira, N.P., Vargas, D.M., Kitajima, E.W., Zaha, A. and Ferreira, H.B. (2012) Echinococcus granulosus antigen B structure: Subunit composition and oligomeric states. PLoS. Negl. Trop. Dis., 6(3): e1551.

33. Felice, G.D., Pini, C., Afferni, C. and Vicari, G. (1986) Purification and partial characterization of the major antigen of Echinococcus granulosus (antigen 5) with monoclonal antibodies. Mol. Biochem. Parasitol., 20(2): 133-142.

34. Lorenzo, C., Salinas, G., Brugnini, A., Wernstedt, C., Hellman, U. and Lez-Sapienza, G.G. (2003) Echinococcus granulosus antigen 5 is closely related to proteases on the trypsin family. Biochem. J., 369(1): 191-198.

35. Carmena, D., Benito, A. and Eraso, E. (2006) Antigens for the immunodiagnosis of Echinococcus granulosus 
infection: An update. Acta. Trop., 98(1): 74-86.

36. Azab, M.E., Bishara, S.A., Helmy, H., Oteifa, N.M., El-Hosein, L.M., Ramzy, R.M. and Ahmed, M.A. (2004) Molecular characterization of Egyptian human and animal E. granulosus isolates by RAPD-PCR technique. J. Egypt. Soc. Parasitol., 34(1): 83-96.

37. Shahnazi, M., Hejazi, H., Salehi, M. and Andalib, A.R. (2011) Molecular characterization of human and animal Echinococcus granulosus isolates in Isfahan, Iran. Acta Trop., 117(1): 47-50.

38. Aaty, H.E.A., Abdel-Hameed, D.M., Alam-Eldin, Y.H.,
El-Shennawy, S.F., Aminou, H.A., Makled, S.S. and Darweesh, S.K. (2012) Molecular genotyping of Echinococcus granulosus in animal and human isolates from Egypt. Act. Trop., 121(2): 125-128.

39. Omar, M., Sultan, K., Haridy, M. and Omran, A. (2013) Prevalence of cystic Echinococcosis in slaughtered ruminants in different abattoirs upper Egypt. Am. J. Anim. Vet. Sci., 8(3): 117-121.

40. Khalifa, N.O., Khater, H.F. and Nassief, M.Z. (2014) Genetic fingerprint of unilocular hydatidosis in Egyptian camels and humans using nested PCR. Pak. Vet. J., 34(4): 522-526.

$* * * * * * * *$ 